\title{
Análise do desempenho da aptidão física relacionada à saúde em escolares praticantes de musculação
}

\author{
Analysis of the performance of health-related physical fitness in school students practicing \\ bodybuilding \\ Análisis del rendimiento de la aptitud física relacionada con la salud en escolares que practican \\ musculación
}

Recebido: 15/05/2021 | Revisado: 20/05/2021 | Aceito: 24/05/2021 | Publicado: 09/06/2021

\author{
Rayssa Camurça Pereira \\ ORCID: https://orcid.org/0000-0001-9340-6103 \\ Instituto Federal de Educação, Ciência e Tecnologia do Ceará, Brasil \\ E-mail: rayssacamurca2203@gmail.com \\ Nilson Vieira Pinto \\ ORCID: https://orcid.org/0000-0001-6548-8586 \\ Instituto Federal de Educação, Ciência e Tecnologia do Ceará, Brasil \\ E-mail: nilsonvieira@ifce.edu.br
}

\begin{abstract}
Resumo
A musculação, embora não seja uma modalidade comumente aplicada na escola, tem se tornado uma atraente estratégia pedagógica capaz de promover a saúde e estimular os seus praticantes a adotarem um estilo de vida saudável. Este estudo teve por objetivo avaliar a aptidão física relacionada à saúde em escolares praticantes de musculação. A amostra foi composta por 132 adolescentes matriculados nas turmas de musculação em uma escola pública do Ceará. A aptidão física relacionada à saúde foi avaliada através da coleta dos dados antropométricos, de composição corporal, dos níveis de flexibilidade, resistência abdominal e aptidão aeróbica, antes e após o período letivo. Os resultados evidenciaram um aumento dos níveis de flexibilidade $(26,55 \pm 9,87$ vs. 27,43 $\pm 9,6)$, resistência abdominal $(34,88 \pm 8,21$ vs. 46,38 $\pm 8,1)$ e resistência aeróbica $(717,9 \pm 68,23$ vs. 734,96 $\pm 62,37)$, entretanto, este incremento não foi capaz de classificá-los fora da zona de risco à saúde cardiovascular. Em conclusão, a prática de musculação ampliou os parâmetros da aptidão física relacionada á saúde, especialmente musculoesquelética, porém, revelou a necessidade de uma melhor sistematização no treinamento aeróbico com vistas à melhora da saúde cardiovascular destes escolares.
\end{abstract}

Palavras-chave: Treinamento de resistência; Aptidão física; Saúde.

\begin{abstract}
Bodybuilding, although it is not a modality commonly applied at school, has become an attractive pedagogical strategy capable of promoting health and encouraging its practitioners to adopt a healthy lifestyle. This study aimed to assess health-related physical fitness in schoolchildren who practice weight training. The sample consisted of 132 adolescents enrolled in bodybuilding classes at a public school in Ceará. Health-related physical fitness was assessed by collecting anthropometric data, body composition, levels of flexibility, abdominal resistance and aerobic fitness, before and after school term. The results showed an increase in the levels of flexibility (26.55 $\pm 9.87 v s .27 .43 \pm 9.6)$, abdominal resistance $(34.88 \pm 8.21 v s .46 .38 \pm 8.1)$ and resistance aerobics $(717.9 \pm 68.23$ vs. $734.96 \pm 62.37)$, however, this increase was not able to classify them outside the risk zone for cardiovascular health. In conclusion, the practice of bodybuilding expanded the parameters of physical fitness related to health, especially musculoskeletal, however, it revealed the need for better systematization in aerobic training in order to improve the cardiovascular health of these students.
\end{abstract}

Keywords: Resistance training; Physical Fitness; Health.

\section{Resumen}

El culturismo, aunque no es una modalidad comúnmente aplicada en la escuela, se ha convertido en una atractiva estrategia pedagógica capaz de promover la salud y alentar a sus practicantes a adoptar un estilo de vida saludable. Este estudio tuvo como objetivo evaluar la aptitud física relacionada con la salud en escolares que practican entrenamiento con pesas. La muestra estuvo conformada por 132 adolescentes inscrito en clases de musculación en una escuela pública de Ceará. La aptitud física relacionada con la salud se evaluó mediante la recopilación de datos antropométricos, composición corporal, niveles de flexibilidad, resistencia abdominal y aptitud aeróbica, antes y después del período escolar. Los resultados mostraron un aumento en los niveles de flexibilidad (26.55 \pm 9.87 vs. 
$27.43 \pm 9.6)$, resistencia abdominal $(34.88 \pm 8.21$ vs. $46.38 \pm 8.1)$ y aeróbicos de resistencia $(717.9 \pm 68.23$ vs.734.96 \pm 62.37 ), sin embargo, esta aumento no pudo clasificarlos fuera de la zona de riesgo para la salud cardiovascular. En conclusión, la práctica del culturismo amplió los parámetros de aptitud física relacionados con la salud, especialmente musculoesquelética, sin embargo, reveló la necesidad de una mejor sistematización en el entrenamiento aeróbico con el fin de mejorar la salud cardiovascular de estos estudiantes.

Palabras clave: Entrenamiento de resistencia; Aptitud física; Salud.

\section{Introdução}

O exercício físico, quando praticado regularmente e sob orientação profissional, desenvolve a aptidão física de seus praticantes e previne o surgimento de inúmeras patologias associadas ao sedentarismo, como obesidade, diabetes e doenças cardiovasculares. A escola representa um dos diversos ambientes favoráveis a esta prática, especialmente durante as aulas de Educação Física, estimulando os escolares a um estilo de vida saudável (Darido \& Souza Júnior, 2007).

É notório que indivíduos sedentários ou com baixo nível de atividade física possuem baixo nível de aptidão física, especialmente a relacionada à saúde. No Brasil, as elevadas prevalências de baixa aptidão física em adolescentes têm sido alvo de inúmeras discussões (Ronque et al., 2007; Souza, 2010; Castro, 2016).

A aptidão física é conceituada como um conjunto de capacidades individuais relacionadas a realização de atividades físicas de forma satisfatória (Castro, 2016). O American College of Sports Medicine (2011) classifica a aptidão física em duas tipologias relacionando-a ao desempenho, quando se refere a performance esportiva, tendo como componentes a agilidade, o equilíbrio, a coordenação, a força, a velocidade e o tempo de reação, ou à saúde, quando se vislumbra a promoção da saúde, tendo como componentes os aspectos morfofuncionais (antropométricos, composição corporal, flexibilidade, força/resistência muscular e aptidão aeróbica).

Uma trajetória pedagógica teórico-prática dos componentes da aptidão física relacionada à saúde possibilita aos adolescentes identificar estes componentes e seus benefícios à saúde, buscando estimular o desenvolvimento de um estilo de vida fisicamente ativo (Bergmann, Araújo, Garlipp, Lorenzi \& Gaya., 2005).

Nas aulas de Educação Física na escola, a prática de esportes tem sido evidenciada em relação aos demais conteúdos curriculares, distanciando os escolares de novas estratégias metodológicas e possibilidades motoras que possam motivar o aluno a prática regular de atividade física (Cardoso, Pereira, Afonso, \& Rocha Junior, 2014).

Neste sentido, a musculação enquanto um conjunto de exercícios físicos, predominantemente analíticos, capazes de manter ou melhorar a aptidão física de seus praticantes (de Macêdo et al., 2014), assume atualmente uma ampla abordagem pedagógica capaz de intervir positivamente na promoção da saúde.

Com a crescente popularidade e consequente aumento do número de adolescentes praticantes de musculação nas academias, torna-se cada vez mais importante a busca pelo entendimento pedagógico que leve a sua aplicabilidade com segurança e à promoção da saúde, especialmente no ambiente escolar.

Diversos estudos têm apontado diversos benefícios aos adolescentes praticantes de treinamento resistido relativos à saúde e ao desempenho físico e esportivo (Behm, Faigenbaum, Falk \& Klentrou, 2008; Ughini, Becker \& Pinto, 2011; Ruas, Brown \& Pinto, 2014; Rosa Santos et al., 2020), em contrapartida, sua aplicabilidade tem sido questionada em relação ao controle de intensidade e volume de treino, capazes de influenciar negativamente nas estruturas passivas como os ossos, ligamentos, cápsulas articulares e tendões (Benedet et al., 2013; Ruas et al., 2014).

Por ser uma modalidade que necessita de alto investimento financeiro na aquisição e na manutenção dos equipamentos é um conteúdo pouco explorado na escola, principalmente se vislumbrarmos a oferta dessa modalidade em instituições públicas de ensino, o que repercute em uma módica análise reflexiva sobre sua abordagem pedagógica e consequentemente na exígua produção científica, geralmente limitada apenas pela adoção do conteúdo teórico sobre a musculação devido a ausência deste ambiente de prática pedagógica. 
Desta forma, este estudo teve como objetivo avaliar a aptidão física relacionada a saúde em escolares praticantes de musculação. Vale ressaltar o caráter singular deste estudo quando se propõe a ampliar o entendimento do caminho pedagógico adequado à promoção da saúde e ao estímulo à adoção de um estilo de vida saudável através da prática de musculação na escola, em destaque, no ensino público, pretendendo discutir as interfaces da ação pedagógica frente a prática de uma modalidade usualmente reconhecida pelo desempenho da performance.

\section{Metodologia}

Este estudo é caracterizado como sendo de delineamento transversal, com abordagem quantitativa, composto por uma amostragem não-probabilística intencional (Pereira et al., 2018), desenvolvido no Instituto Federal de Educação, Ciência e Tecnologia do Ceará - IFCE, campus Fortaleza. Este campus institucional de ensino possui sala de musculação e salas de aula climatizadas e equipadas, com horários específicos destinados ao ensino médio. A amostra foi composta por 132 alunos do Ensino Médio, sendo 83 meninas e 49 meninos, matriculados nas turmas de musculação no período de 2018 a 2019.

Foram incluídos neste estudo alunos com idade entre 13 e 17 anos, que nunca tivessem praticado a modalidade de musculação e com frequência acima de $75 \%$ nas aulas práticas. Foram excluídos aqueles que não cumpriram os critérios de inclusão anteriormente citados, adolescentes grávidas, os que se ausentaram de alguma etapa avaliativa e aqueles que se recusaram a participar, seja de cunho pessoal ou pela recusa dos pais e/ou responsáveis em autorizar a sua participação.

As aulas de musculação aconteceram três vezes por semana, sendo duas práticas intercaladas com uma aula teórica, com duração de 60 minutos cada. No total, foram 180 encontros divididos em três semestres (60 encontros por semestre), incluindo as etapas avaliativas, onde foram avaliados os dados antropométricos, de composição corporal e a aptidão física relacionada a saúde, no início e ao final de cada semestre letivo, realizadas pelo mesmo avaliador, no turno da manhã. Os escolares foram orientados a não praticar exercícios físicos no dia anterior a aplicação dos testes.

A avaliação antropométrica e de composição corporal foi realizada através da mensuração do peso corporal, estatura, índice de massa corporal (IMC), razão circunferência da cintura-quadril (RCQ) e percentual de gordura (\%G).

$\mathrm{O}$ cálculo do IMC foi obtido através do quociente do peso corporal (em $\mathrm{kg}$ ) pelo quadrado da altura em metros $\left(\mathrm{kg} / \mathrm{m}^{2}\right)$. A RCQ foi calculada através do quociente da circunferência da cintura pela circunferência do quadril. A estatura e as circunferências antropométricas foram avaliadas por fita métrica, em centímetros. O peso corporal e o percentual de gordura foram avaliados através de aparelho de bioimpedância (Omron HBF-514C). Estes dados estabelecem associações entre os indicadores antropométricos e de composição corporal e o risco de desenvolver doenças associadas ao excesso de peso.

A aptidão física relacionada à saúde foi avaliada através da realização dos testes de sentar e alcançar, para avaliar os níveis de flexibilidade dos adolescentes, corrida de seis minutos, para avaliar a aptidão aeróbica e o teste Sit-Up para avaliar a resistência abdominal, executadas de acordo com a bateria de testes da PROESP-BR (Gaya \& Gaya, 2016). A análise dos níveis de flexibilidade e da resistência muscular, refletem a saúde musculoesquelética e a avaliação da aptidão aeróbica, pela sua estreita relação com as doenças cardiovasculares, à saúde cardiovascular.

As aulas de musculação tiveram como referencial teórico básico as propostas voltadas à promoção da saúde na Educação Física e como conteúdo prático, a sistematização individual de treinamento baseada nas necessidades físicas diagnosticadas na avaliação física inicial e nos indicadores relacionados ao treinamento de resistência em adolescentes descritos por Benedet et al. (2013), que indicam um programa composto por 8 a 12 exercícios isotônicos de caráter generalizado, utilizando-se inicialmente o peso do corpo, com 8 a 15 repetições, estabelecendo volume moderado e intensidade moderada a baixa e a presença de treinamento cardiovascular e de flexibilidade concomitante.

Os dados antropométricos e de composição corporal foram analisados de acordo com as normas estabelecidas pela World Health Organization (2000). Os resultados dos testes de aptidão física relacionada à saúde foram classificados de acordo 
com as normas do PROESP-BR (Gaya \& Gaya, 2016). Os dados estatísticos foram tabulados inicialmente em uma planilha em excel (windows office 2016) em seguida analisados no software SPSS - Statistical Package for the Social Sciences - versão 20.0, expressos como média e desvio padrão da média. O nível de significância estabelecido foi de p <0,05.

Este estudo seguiu as orientações e determinações fixadas nas Resoluções no. 466, de 12 de dezembro de 2012 e Norma Operacional no. 001/2013, pelo CNS, e encontra-se aprovado pelo Comitê de Ética em Pesquisa do Instituto Federal de Ciência e Tecnologia do Ceará (CEP/IFCE) sob o parecer no. 3.436.618.

\section{Resultados e Discussão}

As avaliações antropométricas e de composição corporal caracterizaram adolescentes eutróficos (IMC entre 18,524,9), muito embora tenham apresentado valores percentuais de gordura corporal elevados (acima de $25 \%$ ) e com risco moderado (RCQ entre 0,71- 0,77) de desenvolver doenças metabólicas causadas pelo acúmulo de gordura corporal (WHO, 2000).

A Tabela 1 evidencia que o programa de exercícios proposto na musculação não promoveu alterações significativas $(\mathrm{p}<0,05)$ nas médias antropométricas e de composição corporal dos adolescentes, ainda que tenha apontado uma leve redução no peso corporal, no IMC e no percentual de gordura quando avaliados em valores absolutos.

Tabela 1: Caracterização antropométrica e de composição corporal da amostra.

\begin{tabular}{ccccc}
\hline & \multicolumn{2}{c}{ Pré-intervenção } & \multicolumn{2}{c}{ Pós-intervenção } \\
Parâmetros & Média & DP & Média & DP \\
\hline Idade (anos) & 16,03 & 1,21 & 16,03 & 1,21 \\
Estatura (metros) & 1,62 & 0,07 & 1,62 & 0,07 \\
Peso (Kg) & 57,81 & 8,93 & 57,57 & 8,83 \\
IMC (kg/m $\mathbf{m}^{\mathbf{2}}$ & 22,09 & 2,99 & 22,02 & 2,94 \\
$\mathbf{\% G}$ & 28,41 & 7,73 & 27,65 & 7,3 \\
$\mathbf{R C Q}$ & 0,75 & 0,05 & 0,75 & 0,05 \\
\hline
\end{tabular}

IMC: índice de massa corporal; \%G: percentual de gordura; RCQ: razão cintura quadril; DP: Desvio padrão; *p<0,05. Fonte: própria.

Embora alguns estudos tenham apontado a musculação como uma estratégia de treinamento físico eficiente na redução ponderal de adolescentes (Velez, Golem \& Arent., 2010; Santos, Guimarães, Santos \& Silva, 2020; Pinto \& Pereira, 2021), este estudo investigou adolescentes que nunca haviam praticado musculação e necessitavam de uma maior supervisão e acompanhamento progressivo das variáveis de treinamento, estabelecendo como referência, um volume moderado e intensidade moderada a baixa, o que provavelmente justifique a inalterabilidade estatística dos parâmetros antropométricos e de composição corporal.

Vale destacar que, estes adolescentes apesar de não terem sido classificados como obesos, apresentam um elevado percentual de gordura e risco moderado de desenvolver doenças associadas ao excesso de gordura corporal, necessitando de uma avaliação do programa de treinamento na musculação com vistas à uma melhor intervenção no controle ponderal desta amostra.

Não obstante, do Nascimento et al. (2021) afirmam que o estilo de vida adotado pelo adolescente pode determinar a sua composição corporal. Este estilo de vida perpassa pelos hábitos alimentares, nível de atividade física, fatores genéticos, nível socioeconômico e fatores emocionais. Essas variáveis intervenientes podem repercutir em uma alta prevalência de sobrepeso/obesidade na adolescência promovendo baixos índices de aptidão física e de capacidade funcional e ampliando a 
possibilidade deste adolescente em desenvolver doenças crônico-degenerativas na vida adulta (Rosa Santos et al., 2020). A busca por um estilo de vida saudável deve estar fundamentada em estratégias de educação em saúde que possam ser estabelecidas entre a família, a escola e o adolescente.

Com base nas normas descritas no manual PROESP-BR (Gaya \& Gaya, 2016), os adolescentes praticantes de musculação foram classificados na zona saudável de aptidão física para a saúde musculoesquelética, apresentando médias de flexibilidade e resistência abdominal acima dos valores de corte. Contudo, estão inseridos na zona de risco à saúde cardiovascular, caracterizando uma amostra com baixa aptidão aeróbica (Tabela 2).

Tabela 2: Aptidão Física relacionada à saúde.

\begin{tabular}{ccccc}
\hline \multirow{2}{*}{ Parâmetros } & \multicolumn{2}{c}{ Pré-intervenção } & \multicolumn{2}{c}{ Pós-intervenção } \\
Média & DP & Média & DP \\
\hline Flexibilidade (cm) & 26,55 & 9,87 & $27,43^{*}$ & 9,6 \\
RAB (rep) & 34,88 & 8,21 & $46,38^{*}$ & 8,1 \\
RAERO (m) & 717,9 & 68,23 & $734,96^{*}$ & 62,37 \\
\hline
\end{tabular}

RAB: Resistência abdominal; RAERO: Resistência aeróbica; DP: Desvio padrão; cm: centímetros; rep: repetições; m: metros; *p<0,05. Fonte: própria.

Nesta trajetória, se faz importante destacar que as doenças cardiovasculares são consideradas a principal causa de mortalidade mundial e estão relacionadas com o estilo de vida adotado desde a adolescência (Lancarotte, Nobre, Zanetta, \& Polydoro, 2010). Identificamos neste estudo uma amostra de adolescentes que mesmo praticando a musculação apresentam médias elevadas nos parâmetros antropométricos e de composição corporal que podem, em associação a baixa aptidão aeróbica, predispor estes escolares ao desenvolvimento de doenças crônico-degenerativas na vida adulta. Reitera-se então, a necessidade de estratégias pedagógicas que possam intervir na mudança do estilo de vida destes adolescentes.

Vale ressaltar que, a prática sistematizada de musculação promoveu uma melhora significativa em todos os parâmetros de aptidão física relacionada à saúde investigados neste estudo, entretanto, não ampliou de forma expressiva a aptidão aeróbica desses adolescentes capaz de inseri-los na zona saudável de saúde cardiovascular. Todavia, é importante esclarecer que embora o programa de exercícios proposto na musculação oportunizasse o treinamento cardiovascular, as estratégias pedagógicas utilizadas nas aulas práticas enfatizavam o desenvolvimento da saúde musculoesquelética. Esta reflexão sugere que, para esta amostra, a inserção de um treino aeróbico concomitante poderá melhorar a saúde cardiovascular e auxiliar no controle ponderal destes adolescentes.

Stricker, Faigenbaum e Mccambridge (2020) apontam que o treinamento de resistência promove a melhora da saúde e do condicionamento físico, promovendo ainda uma "alfabetização física" quando realizado com ênfase na técnica correta e sob supervisão adequada. Kennedy et al., (2018) destacaram que o treinamento de resistência é capaz de gerar uma mudança de comportamento nos escolares possibilitando uma maior adesão nas aulas de educação física. Pinto e Pereira (2021) acrescentam que o treinamento de resistência inserido nas aulas de educação física escolar possibilita procedimentos educacionais importantes para promover um estilo de vida saudável para além dos anos escolares.

Desta forma, os resultados deste estudo apontam que prática da musculação inserida na escola como uma possibilidade pedagógica nas aulas de Educação Física, quando sistematizada e supervisionada adequadamente, podem promover a melhora da aptidão física relacionada à saúde musculoesquelética dos escolares e estimular a adoção de um estilo de vida saudável. 


\section{Considerações Finais}

Os adolescentes praticantes de musculação avaliados neste estudo apresentaram níveis elevados de gordura corporal, relacionando-os a um risco moderado de desenvolver doenças crônico-degenerativas. A intervenção pedagógica com a musculação não foi capaz de promover uma redução significativa na composição corporal que deslocasse estes escolares da zona de risco à saúde.

A prática de musculação promoveu a melhora de todos os parâmetros da aptidão física relacionada á saúde, especialmente, musculoesquelética, entretanto não conseguiu ampliar a aptidão aeróbica destes adolescentes de forma suficiente que pudesse classificá-los fora da zona de risco à saúde cardiovascular.

Todavia, é importante evidenciar que o treinamento de resistência tem como foco o desempenho da saúde musculoesquelética, entretanto, estes resultados revelaram a necessidade de ampliação do componente aeróbico no treinamento de musculação e/ou inserção de treino aeróbico concomitante, com vistas a redução ponderal e a melhora da aptidão cardiorrespiratória destes adolescentes.

Em adição, faz-se oportuno possibilitar novas estratégias pedagógicas nas aulas de educação física escolar capazes de estimular um estilo de vida mais saudável, e assim, prevenir o surgimento de doenças crônico-degenerativas no futuro.

A musculação ainda é uma abordagem pedagógica pouco explorada na escola. Contudo, a inserção do treinamento de resistência nas aulas de educação física escolar, além de ampliar a motivação dos escolares, pode promover a melhora dos componentes da aptidão física relacionada à saúde e prevenir possíveis doenças crônico-degenerativas na vida adulta.

Este estudo poderá contribuir na reflexão e na estruturação metodológica do treinamento resistido na escola capazes de influenciar no desempenho da aptidão física e na promoção da saúde dos escolares.

\section{Referências}

American College of Sports Medicine. (2011). Manual do ACSM para avaliação da aptidão física relacionada à saúde. Grupo Gen - Guanabara Koogan.

Behm, D. G., Faigenbaum, A. D., Falk, B., \& Klentrou, P. (2008). Canadian Society for Exercise Physiology position paper: resistance training in children and adolescents. Applied physiology, nutrition, and metabolism, 33(3), 547-561.

Benedet, J., Freddi, J. C., Luciano, A. P., Almeida, F. D. S., Silva, G. L. D., Hinnig, P. D. F., \& Adami, F. (2013). Treinamento resistido para crianças e adolescentes. ABCS health sci. 38(1):40-46.

Bergmann, G. G., Araújo, M. L. B. D., Garlipp, D. C., Lorenzi, T. D. C., \& Gaya, A. (2005). Alteração anual no crescimento e na aptidão física relacionada à saúde de escolares. Rev Bras Cineantropom Desempenho Hum, 7(2), 55-61.

Castro, F. J. S. D. (2016). Aptidão física relacionada à saúde e desempenho acadêmico de adolescentes. Dissertação de Mestrado, Universidade Federal de Sergipe, Se, Brasil.

Cardoso, M. A., Pereira, F. M., Afonso, M. D. R., \& Rocha Junior, I. C. D. (2014). Educação física no ensino médio: desenvolvimento de conceitos e da aptidão física relacionados à saúde. Revista Brasileira de Educação Física e Esporte, 28(1), 147-161.

Darido, S. C., \& De Souza Jr, O. M. (2007). Para ensinar educação física. Papirus Editora.

de Macêdo, P. P., de Souza, F. J. R., Junior, T. D. A. A., Letieri, R. V., da Silva Neto, L. V., Filho, N. T., \& Junior, J. A. D. F. P. A. (2014). A musculação como conteúdo nas aulas de educação física escolar. Coleção Pesquisa em Educação Física, 13(4), 15-22.

Gaya, A., \& Gaya, A. R. (2016). Projeto esporte Brasil: manual de testes e avaliação: UFRGS, 1-26.

Kennedy, S. G., Smith, J. J., Morgan, P. J., Peralta, L. R., Hilland, T. A., Eather, N., \& Lubans, D. R. (2018). Implementing resistance training in secondary schools: a cluster randomized controlled trial. Medicine \& Science in Sports \& Exercise, 50(1), 62-72

Lancarotte, I., Nobre, M. R., Zanetta, R., \& Polydoro, M. (2010). Estilo de vida e saúde cardiovascular em adolescentes de escolas do município de São Paulo. Arquivos Brasileiros de Cardiologia, 95(1), 61-69.

do Nascimento, M. B., de Jesus Porto, M., de Souza, J. P., de Souza, M. D. F. S., Araujo, A. M. B., de Andrade, W. B., \& Silva, S. R. S. Obesidade na adolescência: um perfil traçado atualmente através de revisão narrativa. Research, Society and Development, 10(1), e26710111857-e26710111857.

Pereira, A. S., et al. (2018). Metodologia da pesquisa científica. [e-book]. Santa Maria. Ed. UAB/NTE/UFSM. <https://repositorio.ufsm.br/bitstream/handle/1/15824/Lic_Computacao_Metodologia-Pesquisa-Cientifica.pdf?sequence=1> 
Research, Society and Development, v. 10, n. 6, e54110616111, 2021

(CC BY 4.0) | ISSN 2525-3409 | DOI: http://dx.doi.org/10.33448/rsd-v10i6.16111

Pinto, N. V., \& Pereira, R. C. (2021). Treinamento de resistência em escolares: Uma Revisão Integrativa. Research, Society and Development, 10(3), e54510313697.

Ronque, E. R. V., Cyrino, E. S., Dórea, V., Serassuelo Júnior, H., Galdi, E. H. G., \& Arruda, M. D. (2007). Diagnóstico da aptidão física em escolares de alto nível socioeconômico: avaliação referenciada por critérios de saúde. Revista Brasileira de Medicina do Esporte, 13(2), 71-76.

Rosa Santos, L. D., Araujo, S. S. D., Vieira, E. F. D. S., Estevam, C. D. S., Santos, J. L. D., Wichi, R. B., \& Marçal, A. C. (2020). Effects of 12 weeks of resistance training on cardiovascular risk factors in school adolescents. Medicina, 56(5), 220.

Ruas, C. V., Brown, L. E., \& Pinto, R. S. (2014). Treinamento de força para crianças e adolescentes: adaptações, riscos e linhas de orientação. Brazilian Journal of Motor Behavior (BJMB). Porto Alegre/RS, Brasil, 8(1), 1-9.

Santos, G. de O., Guimarães, A. H. A., Santos, L. M., \& Silva, S. L. da. (2020). The influence of weight training on body composition and waist-to-hip ratio. Research, Society and Development, 9(10), e3669108782. https://doi.org/10.33448/rsd-v9i10.8782

Souza, E. A. D. (2010). Associação da prática de atividade física com a aptidão física relacionada á saúde em escolares da cidade de Fortaleza. Dissertação de mestrado, Universidade de Brasília, Brasília, Brasil.

Stricker, P. R., Faigenbaum, A. D., \& McCambridge, T. M. (2020). Resistance training for children and adolescents. Pediatrics, 145(6).

Ughini, C. C., Becker, C., \& Pinto, R. S. (2011). Treinamento de força em crianças: segurança, benefícios e recomendações. Conexões, 9(2), 177-197.

Velez, A., Golem, D. L., \& Arent, S. M. (2010). The impact of a 12-week resistance training program on strength, body composition, and self-concept of Hispanic adolescents. The Journal of Strength \& Conditioning Research, 24(4), 1065-1073.

WHO. (2000). Obesity: preventing and managing the global epidemic. Report of a World Health Organization Consultation. Geneva: World Health Organization. p. 256. WHO Obesity Technical Report Series, n. 284. 